\title{
COMPARACIÓN DE LA ACTIVIDAD NOCTURNA DE POBLACIONES DE Artibeus obscurus (Chiroptera: Phyllostomidae) QUE HABITAN EN CONSTRUCCIONES HUMANAS Y CUEVAS, EN EL DEPARTAMENTO DE SUCRE, COLOMBIA
}

\author{
COMPARISON OF NIGHTTIME ACTIVITIES OF POPULATIONS OF Artibeus \\ obscurus (Chiroptera: Phyllostomidae) THAT INHABITS IN HUMAN \\ CONSTRUCTIONS AND CAVES, IN THE DEPARTMENT OF SUCRE, COLOMBIA
}

\author{
SAMPEDRO-M, ALCIDES ${ }^{1}$. Doctor en Ciencias Biológicas, MENDOZA-D, \\ KAREN. Estudiante de Biología ${ }^{1}$. \\ ${ }^{1}$ Grupo de Biodiversidad Tropical, Universidad de Sucre. Dpto. de Biología. \\ *Correspondencia: asampedro2002@yahoo.es tulipan2430@hotmail.com
}

\section{Resumen}

Durante doce meses (mayo 2008-mayo 2009) se realizaron 15 muestreos con redes de niebla de 12x2 en las cercanías de diversas construcciones humanas (viviendas, empresas, universidad) del municipio de Sincelejo, departamento de Sucre y 10 muestreos en las cercanías de la cueva del municipio de San Antonio de Palmito y de la cueva del centro ecoturístico Roca Madre, en Toluviejo, ambas en el departamento de Sucre. Los muestreos se hicieron en el período comprendido entre las 18:00 y las 06:00. Las redes eran revisadas cada $1 / 2$ hora y se clasificaba cada ejemplar, se anotaba la hora y se determinaba el sexo y la condición reproductiva. Se consideraron 4 períodos de actividad nocturna, entre las 18:00 y las 20:59; entre las $21: 00$ y las 23:59; entre 00:00 y las 02:59 y entre las 03:00 y las 06:00. Se observaron diferencias en los horarios de actividad de los individuos de los dos tipos de refugio en relación con el período (lluvia y seca) y el sexo, lo que posiblemente esté relacionado con las diferentes condiciones de competencia por el espacio en cada caso, así como con las necesidades energéticas de la especie. Al analizar la actividad para las hembras con diferente condición reproductiva se observó un comportamiento diferente para cada grupo y a la vez similar para los dos tipos de refugio, lo que pudiera constituir un mecanismo de protección de las crías, ya que nunca permanecen solas en el interior de los refugios.

Palabras clave: Artibeus obscurus, actividad nocturna, cuidados parentales, refugio diurno. 


\section{Abstract}

During twelve months (May 2008-May 2009) fifteen samplings with fog nets (12x2) were made in the neighborhoods of diverse human constructions (houses, companies, university) of the municipality of Sincelejo, department of Sucre and ten samplings in the neighborhoods of the cave of the municipality of San Antonio de Palmito and in the cave of the eco-tourist center Roca Madre in Toluviejo, both in the department of Sucre. The samplings were made in the period between 18:00 and the 06:00. The nets were checked each 1/2 hour and each individual was classified, the hour was noted down and sex and the reproductive condition were determined. Four periods of nighttime activities were considered, between 18:00 and 20:59; 21:00 and 23:59; 00:00 and 2:59 and 03:00 and 06:00. Differences in the activity schedules of the individuals of both types of refuge were observed in relation with the period (dry and rain) and sex. This might be related to the different conditions of competition for the area control in each case, as well as with the energy-giving necessities of the species. When analyzing the activity of females with different reproductive condition a different behavior was observed in each group and simultaneously similar for both types of refuge, which could constitute a mechanism of protection for the young, since they never remain alone inside the refuges.

Key words: Artibeus obscurus, night time activities, parental care, daytime refuge.

\section{Introducción}

Las investigaciones relacionadas con la actividad nocturna de los murciélagos han planteado diferencias entre las especies y entre sexos en relación con los horarios de salida y regreso a los refugios (SILVA, 1979; SAMPEDRO et al, 2007) y se ha considerado que este hecho desempeña un papel importante en la disminución de la competencia entre los organismos y que también está relacionado con las exigencias energéticas de cada especie. Como los murciélagos de una misma especie pueden ocupar diferentes tipos de refugios diurnos, la actividad nocturna también pudiera variar.

No se observa en la literatura mucha información con respecto a la estrategia relacionada con el cuidado de las crías durante la actividad nocturna. Durante 
el período de forrajeo las crías que quedan en el refugio pudieran encontrarse desamparadas.

HILL y SMITH (1984), describen dos estrategias de atención a las crías durante la actividad nocturna. Una la efectúan aquellas especies que no son capaces de llevar a sus hijos durante el forrajeo nocturno y que los dejan agrupados para no perder temperatura corporal y cuidados por "niñeras"; y las que pueden llevarlos durante el vuelo y por tanto las cuidan por sí mismas (MUÑOZ, 2001; SAMPEDRO et al., 2008a). Actualmente se ha observado algunas variantes, como sucede con algunas especies insectívoras que regresan rápido al refugio y por tanto las crías permanecen solas muy poco tiempo y en lugares por lo general inaccesibles (GUNDLACH, 1877; SILVA, 1979; NOWAK, 1997; MUÑOZ, 2001; AGUIRRE, 2002; SAMPEDRO et al., 2007; SAMPEDRO et al., 2008b). A partir de estos elementos, en el presente artículo se pretende determinar si existen diferencias en la actividad nocturna y la estrategia de cuidado de las crías durante esa actividad, entre poblaciones de Artibeus obscurus, que habitan en dos tipos de refugios, construcciones humanas y cuevas naturales en el departamento de Sucre, Colombia.

\section{Materiales y métodos}

El estudio se llevó a cabo entre los meses de mayo de 2008 y mayo de 2009. Se efectuaron 15 muestreos con redes de niebla de $12 \times 2 \mathrm{~m}$, en las cercanías (5 m) de diversas construcciones humanas (viviendas, empresas, universidad de Sucre) del municipio de Sincelejo, departamento de Sucre, se llevaron a cabo también 10 muestreos en las cercanías $(5 \mathrm{~m})$ de la cueva del municipio de San Antonio de Palmito (09 20'14.7" N; $075^{\circ} 32^{\prime} 50.5^{\prime \prime}$ O) y de la cueva del centro ecoturístico Roca Madre (09³0' 50.5” N; 075²3' 36.6” O) (Figs. 1 y 2), en el municipio de Toluviejo, ambas áreas ubicadas en el departamento de Sucre. 
Los muestreos se realizaron en el período comprendido entre las 18:00 y las 06:00 horas utilizando redes de niebla de $12 \times 2$, que eran revisadas cada $1 / 2$ hora (Fig. 2).

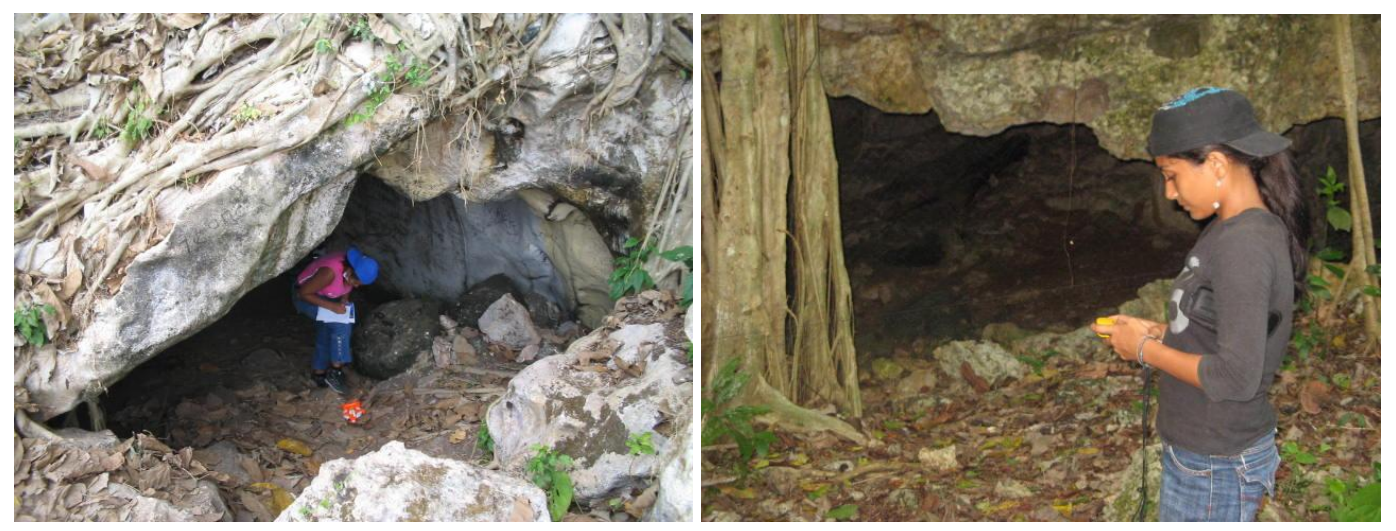

Figura 1. Entrada principal de las cuevas de San Antonio de Palmito (izquierda) y del Centro de Ecoturismo Roca Madre Aventura en Toluviejo.

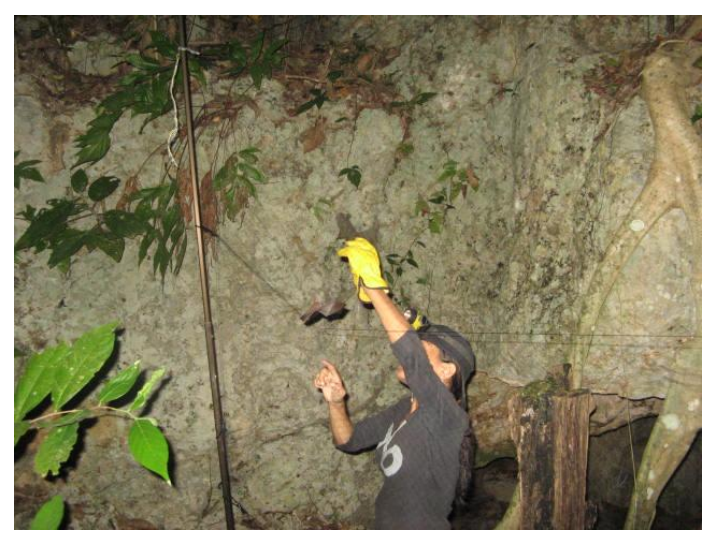

Figura. 2. Revisión de redes de niebla cada $1 \frac{1}{2}$ hora, entre las $18: 00$ y las 06:00

En el momento de la captura se identificaba cada ejemplar (Fig.3), se anotaba la hora, el sexo y la condición reproductiva, indicando para los machos: reproductores y no reproductores, mientras que para las hembras se dividieron en gestantes, lactantes y no reproductoras, según la metodología de SILVA (1979). 


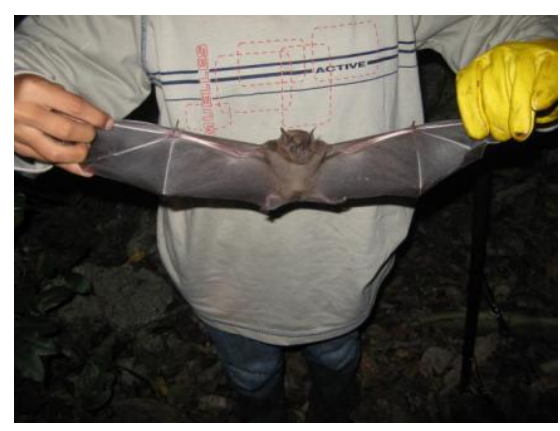

Figura 3. Ejemplar de Artibeus obscurus momentos después de su captura

Se realizaron dos muestreos con jama en el interior de la cueva de San Antonio de Palmito, uno a las 03:30 y el otro a las 05:00. En ambos casos se trató de un grupo compacto de adultos y juveniles que se encontraban en el sito de reposo (Fig. 4). Los murciélagos capturados fueron liberados inmediatamente después de obtenidos los datos.

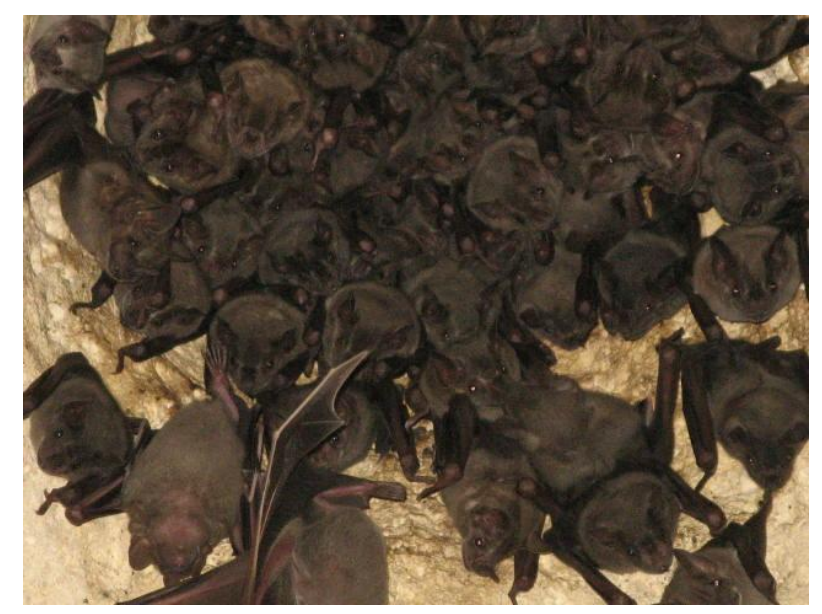

Figura 4. Grupo compacto de adultos y juveniles de Artibeus obscrus en el sitio de reposo, en el interior de la cueva de San Antonio de Palmito

Para los análisis se agruparon los datos en 4 períodos de actividad nocturna, entre las 18:00 y las 20:59 horas; entre las 21:00 y las 23:59 horas; entre 00:00 y las 02:59 horas, y entre las 03:00 y las 06:00 horas. La comparación de la frecuencia de individuos de ambos sexos, entre períodos de seca y lluvia y entre animales con diferente condición reproductiva, para las colectas en ambos tipos de refugio se hizo a través de una Tabla de Contingencia de $2 \times 2$. (Zar, 1996) 


\section{Resultados}

En la Tabla 1 aparece el número de individuos de los diferentes sexos, condición reproductiva y período del año, capturados en las cercanías de sus refugios (construcciones humanas y cuevas), durante su actividad nocturna.

En la Fig. 5 puede apreciarse la frecuencia de captura de murciélagos en las cercanías a construcciones humanas y en la Fig. 6, la frecuencia de captura con respecto a las cuevas naturales. En el primer caso se observa una mayor frecuencia de individuos después de la media noche, mientras que los que habitan las cuevas parecen presentar mayor actividad en las primeras horas de la noche. No obstante, para ambos casos se observa cierta actividad durante toda la jornada nocturna.

Tabla 1. Número de individuos de Artibeus obscurus capturados en las cercanías a sus refugios durante su actividad nocturna.

\begin{tabular}{|c|c|c|c|c|c|c|c|c|}
\hline \multirow{2}{*}{$\begin{array}{l}\text { Hora de } \\
\text { captura }\end{array}$} & \multirow{2}{*}{$\mathbf{N}$} & \multicolumn{2}{|c|}{ Período } & \multicolumn{2}{|c|}{ Sexo } & \multicolumn{3}{|c|}{$\begin{array}{c}\text { Condición reproductiva } \\
\text { de las hembras }\end{array}$} \\
\hline & & Lluvia & Seca & Hembras & Machos & G & $\mathbf{L}$ & NR \\
\hline \multicolumn{9}{|c|}{ CONSTRUCCIONES HUMANAS } \\
\hline 18:00-20:59 & 18 & 16 & 3 & 16 & 4 & 1 & 5 & 10 \\
\hline 21:00-23:59 & 22 & 14 & 1 & 14 & 2 & 0 & 8 & 6 \\
\hline 00:00-02:59 & 48 & 26 & 20 & 35 & 15 & 0 & 25 & 10 \\
\hline 03:00:06:00 & 44 & 29 & 23 & 30 & 16 & 27 & 2 & 1 \\
\hline Total & 132 & 85 & 47 & 95 & 37 & 28 & 40 & 27 \\
\hline \multicolumn{9}{|c|}{ CUEVAS } \\
\hline 18:00-20:59 & 24 & 18 & 15 & 18 & 7 & 0 & 16 & 8 \\
\hline 21:00-23:59 & 15 & 10 & 9 & 12 & 3 & 0 & 6 & 6 \\
\hline 00:00-02:59 & 16 & 5 & 2 & 12 & 3 & 0 & 5 & 7 \\
\hline 03:00:06:00 & 16 & 9 & 3 & 13 & 3 & 12 & 0 & 1 \\
\hline Total & 71 & 42 & 29 & 55 & 16 & 12 & 21 & 22 \\
\hline
\end{tabular}


Al considerar la actividad nocturna de los murciélagos que habitan en construcciones humanas, durante el período de lluvia y seca (Fig. 7) se observaron diferencias altamente significativas $\left(X^{2}=11.66, p<0.001, G L=3\right)$, ya que en el período lluvioso existe mayor actividad durante la primera parte de la noche y en el período seco la mayor actividad se registra después de la media noche. En el caso de los murciélagos que habitan en las cuevas (Fig. 8), no se presentaron diferencias significativas para la actividad en los distintos períodos $\left(X^{2}=2.31, p>0.05, G L=3\right)$.

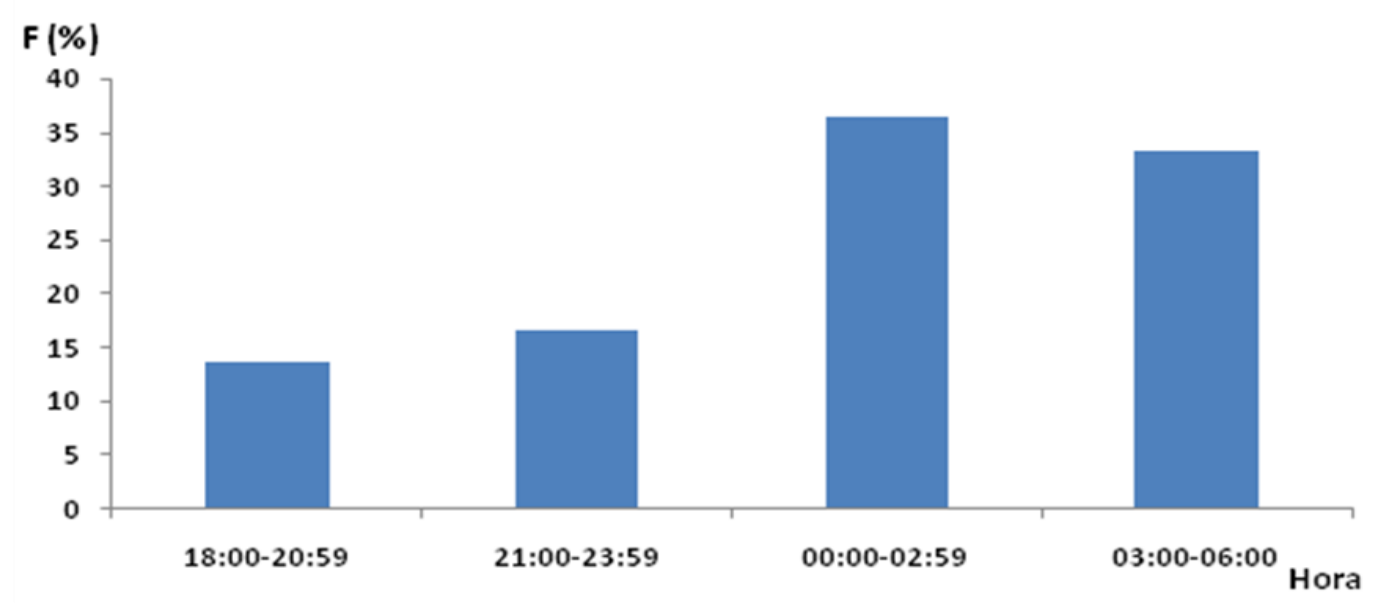

Figura 5. Frecuencia de captura $(F)$ de Artibeus obscurus en las cercanías a construcciones humanas en el municipio de Sincelejo, Sucre.

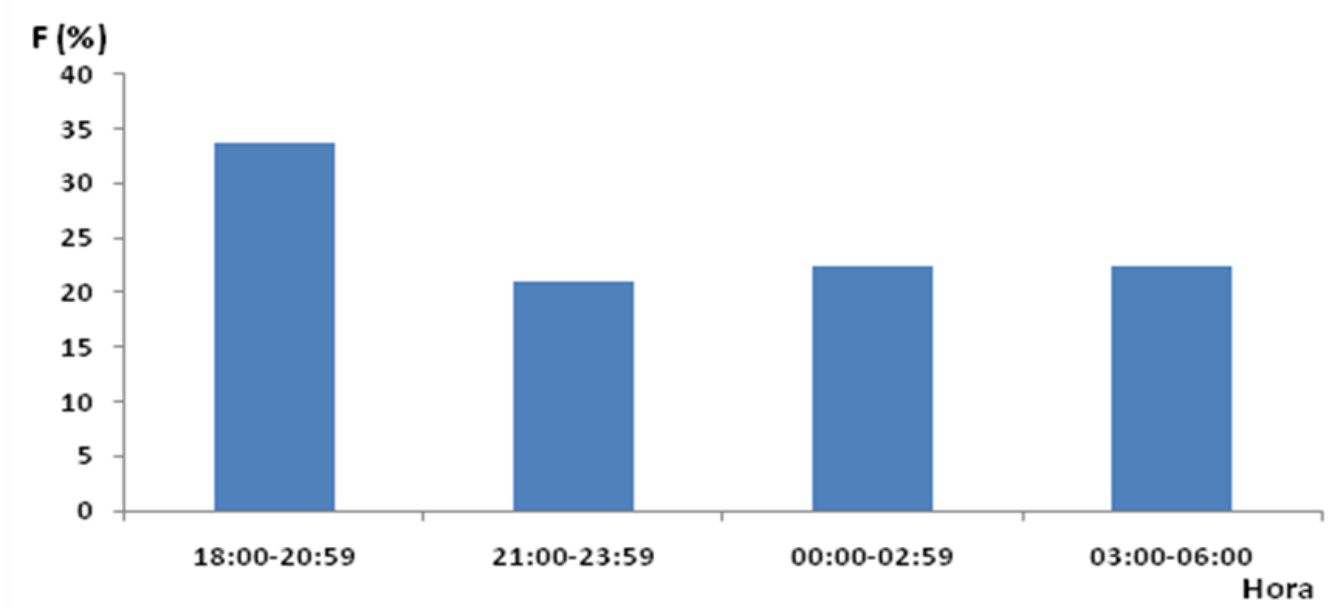

Figura 6. Frecuencioa de captura (F) de Artibeus obscurus en las cercanías a cuevas naturales en los municipios de San Antonio de Palmito y Toluviejo, Sucre. 
En relación con los sexos no se presentaron diferencias significativas al comparar con los horarios de actividad de las dos poblaciones estudiadas (Figs. 9 y 10) $\left(X^{2}=3.69, p>0.05, G L=3 ; X^{2}=0.67, p>0.05, G L=3\right)$; sin embargo, al analizar la actividad nocturna de las hembras de acuerdo a su condición reproductiva se aprecian diferencias altamente significativas (Fig. 11 y 12) $\left(X^{2}=\right.$ 86.16, $p>0.001, G L=6) ; X^{2}=50.42, p<0.001, G L=6$ ). En este caso, las hembras lactantes y las no reproductivas de ambos tipos de refugio, se mantienen activas durante toda la noche, excepto entre las 03:00 y las 06:00, mientras que las gestantes solo tienen actividad durante las últimas horas de la noche.

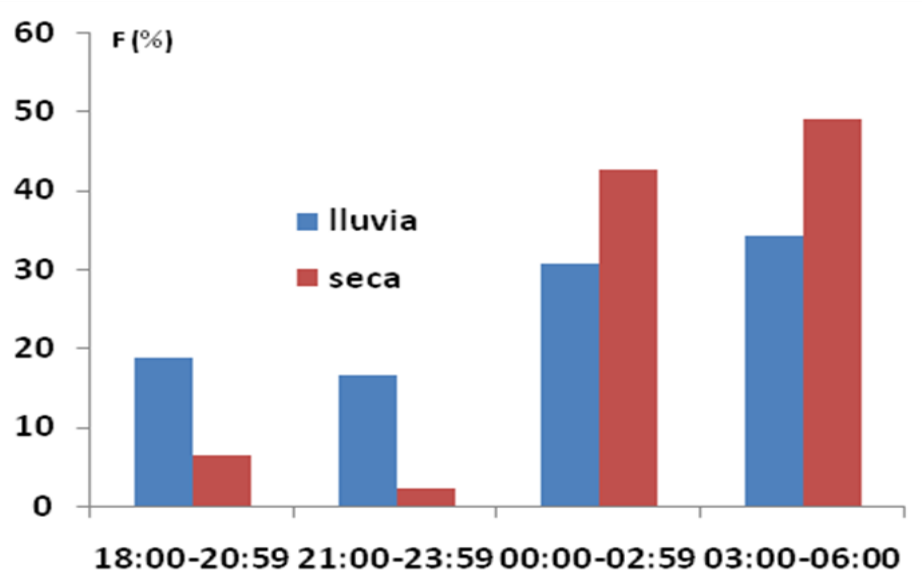

Figura 7. Frecuencia de captura (F) de Artibeus obscurus en las cercanías a construcciones humanas en el municipio de Sincelejo, Sucre, durante los períodos de lluvia y seca.

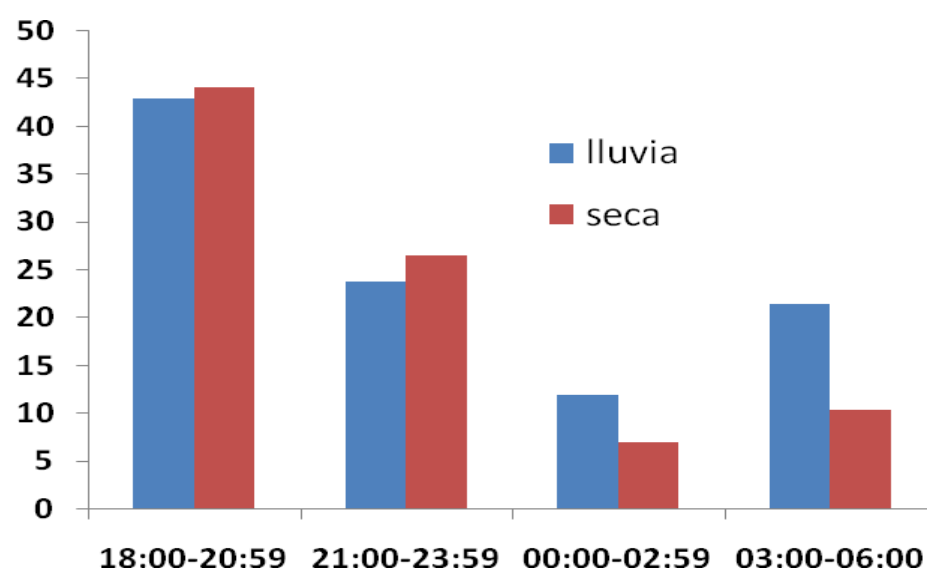

Figura 8. Frecuencia de captura (F) de Artibeus obscurus en las cercanías a cuevas naturales en los municipios de San Antonio de Palmito y Toluviejo, Sucre, durante los períodos de lluvia y seca. 


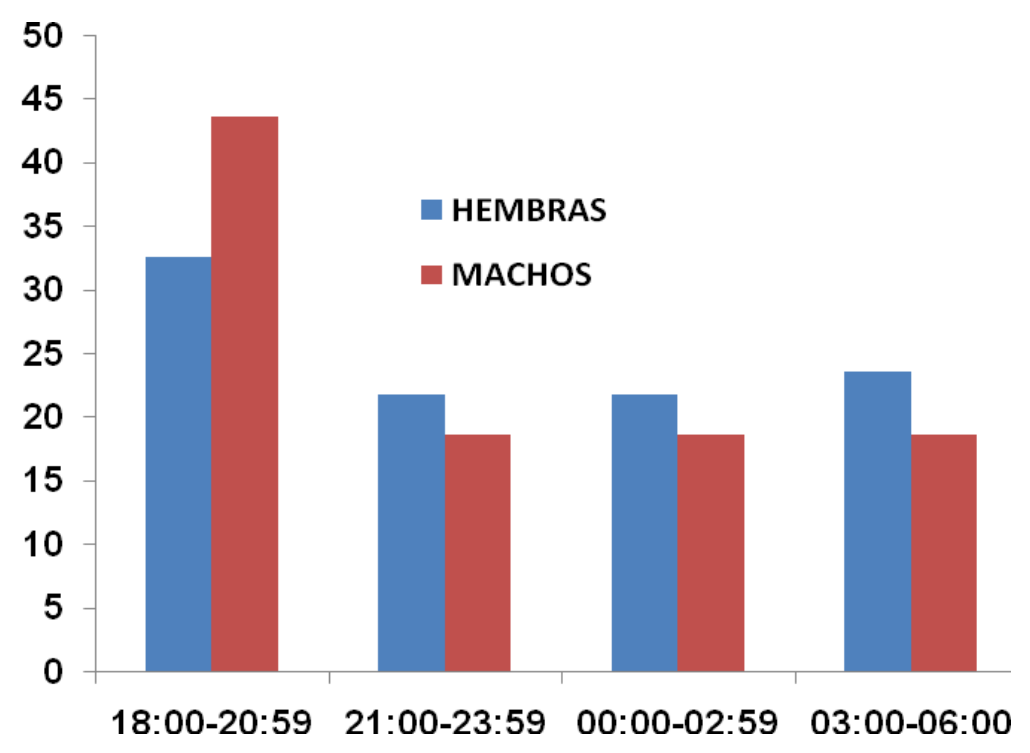

Figura 9. Frecuencia de captura (F) de Artibeus obscurus de ambos sexos en las cercanías a construcciones humanas en el municipio de Sincelejo, Sucre.

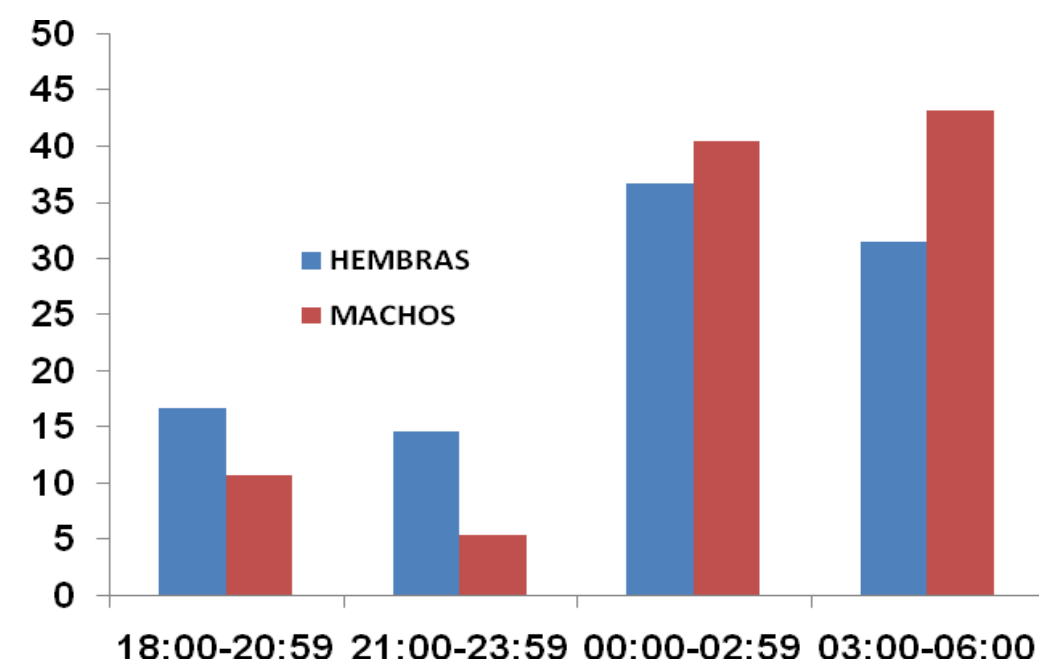

Figura 10. Frecuencia de captura (F) de Artibeus obscurus de ambos sexos en las cercanías a cuevas naturales en los municipios de San Antonio de Palmito y Toluviejo, Sucre. 


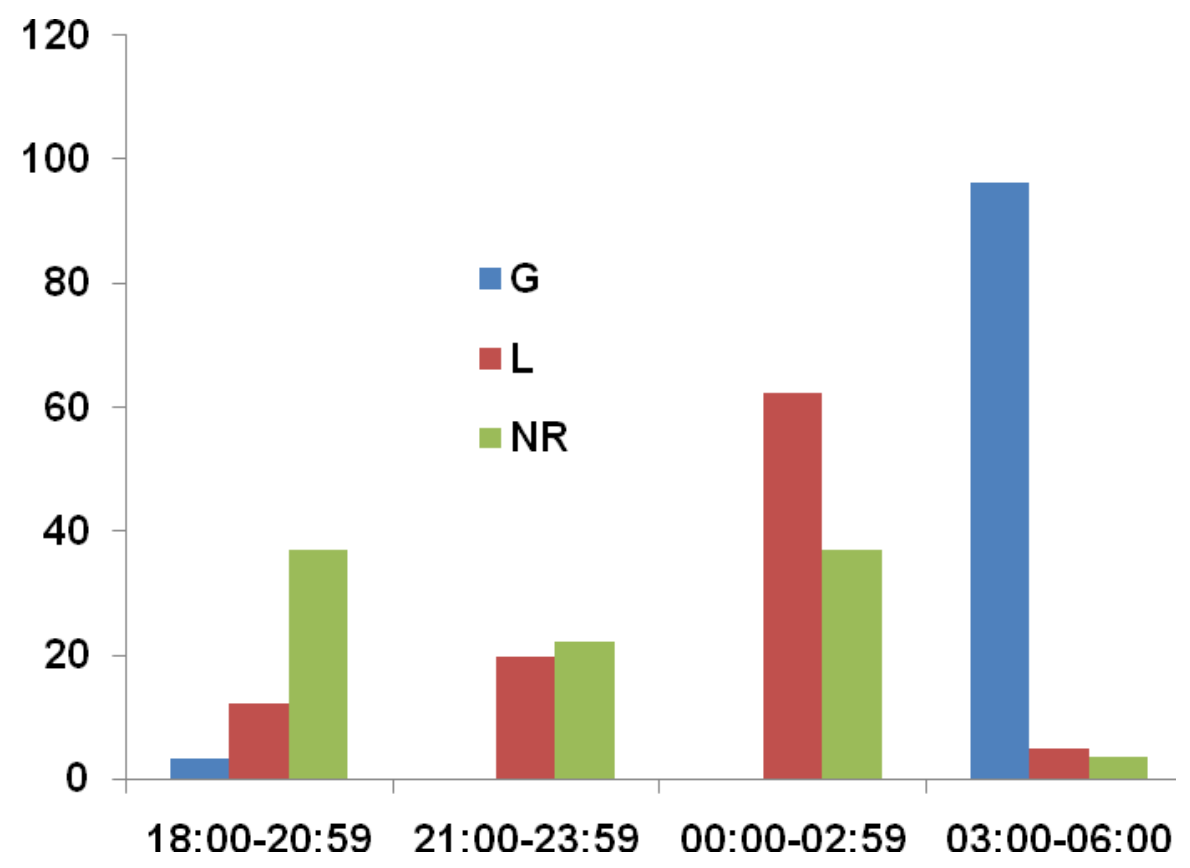

Figura 11. Frecuencia de captura (F) de hembras de Artibeus obscurus con diferente condición reproductiva, en las cercanías a construcciones humanas en el municipio de Sincelejo, Sucre.

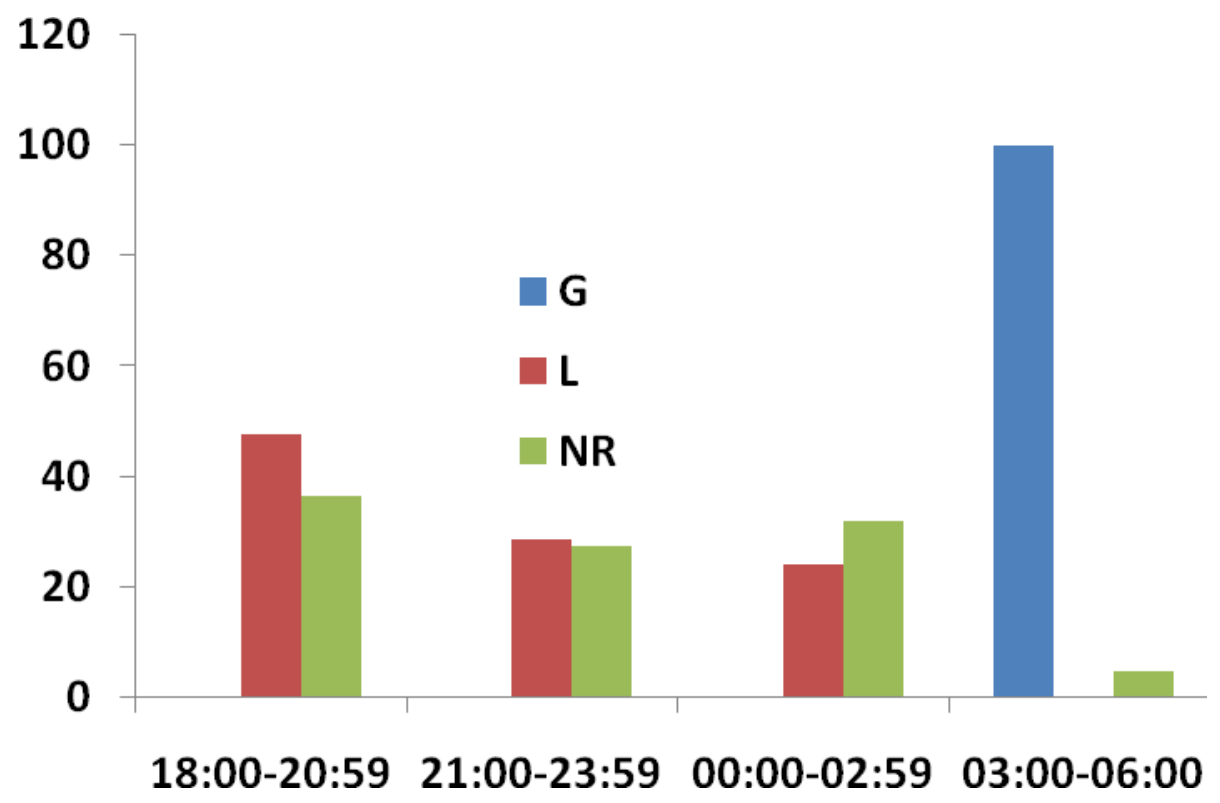

Figura 12. Frecuencia de captura (F) de hembras de Artibeus obscurus con diferente condición reproductiva, en las cercanías a cuevas naturales en los municipios de San Antonio de Palmito y Toluviejo, Sucre. 
El muestreo directo mediante jama en la cueva de San Antonio de Palmito en dos horarios diferentes (Tabla 2), mostró que a las 01:30 el grupo de adultos y juveniles en su mayor parte estaba integrado por hembras gestantes, mientras que a las 05:00 los adultos eran fundamentalmente hembras lactantes y no reproductivas. Los machos aparecen en un número muy bajo y reproductivos no había ninguno. Los juveniles se presentaron en número similar en ambos muestreos.

Tabla 2. Número de individuos adultos de ambos sexos y juveniles capturados en dos muestreos realizados en diferente horario en los sitios de reposo de Artibeus obscurus, en la cueva de San Antonio de Palmito. ( $\mathrm{N}$ = número de individuos).

\begin{tabular}{|c|c|c|}
\hline Resultados del muestreo & $\mathbf{0 1 : 3 0}$ & $\mathbf{0 5 : 0 0}$ \\
$\mathrm{N}$ & 45 & 52 \\
Hembras gestantes & 29 & 3 \\
Hembras lactantes & 7 & 26 \\
Hembras no reproductivas & 5 & 20 \\
Machos reproductivos & 0 & 0 \\
Machos no reproductivos & 1 & 3 \\
juveniles & 17 & 21 \\
\hline
\end{tabular}

\section{Discusión}

El análisis de las condiciones en que se desempeñan los murciélagos cuando habitan en cuevas y cuando están en construcciones humanas, puede ofrecer alguna explicación con respecto a su actividad nocturna. En las construcciones humanas la salida al exterior generalmente es la misma para todos los individuos, lo que deberá traer como consecuencia una cierta competencia en el horario de salida. En el caso de las cuevas generalmente no se observa 
esto, sino que los animales disponen de diferentes sitios de salida, a menudo de mayor tamaño, y por tanto no compiten necesariamente por el horario para comenzar su actividad nocturna. Esto podría explicar la razón de que Artibeus obscurus cuando habita en construcciones humanas muestra tendencia a salir en las últimas horas de la madrugada, ya que las especies insectívoras con las que conviven se ven obligadas a salir en las primeras horas, que es cuando los insectos se encuentran en mayor actividad (DOLSA y ALBARRÁN, 1998). Sin embargo, cuando esta especie habita en cuevas, se vio clara su tendencia a salir en las primeras horas de la noche. El período del año que se trate parece influir en esto, ya que durante el período de lluvia los animales de ambos tipos de refugio mantienen cierta actividad durante toda la jornada nocturna. Es posible que la disminución de la temperatura durante el período lluvioso en esta región, unido a la mayor disponibilidad del recurso alimentario, frutas en este caso (MUÑOZ, 2001), determine que una gran cantidad de animales se mantenga activos durante toda la noche. De todas maneras, los resultados parecen confirmar las aseveraciones acerca de que la utilización de diferentes horarios para realizar su actividad nocturna, puede representar una disminución en la competencia, sobre todo en los que viven en refugios con pocas y estrechas salidas (SILVA, 1979; SAMPEDRO et al, 2007).

El análisis de la actividad en relación con los sexos ofrece el mismo resultado. Ambos sexos que habitan en construcciones humanas utilizan las últimas horas de la noche para salir y los de cuevas, las primeras horas, aunque en los dos tipos de refugio se mantiene una cierta actividad durante toda la noche. Sin embargo, cuando se observa la actividad de las hembras con diferente condición reproductiva, la actividad varía completamente y es igual para los animales de ambos refugios. Las gestantes solo son activas al final de la noche y las lactantes y no reproductivas durante toda la noche, excepto al final, lo que pudiera significar que se trata de un mecanismo de protección de las crías durante la actividad nocturna, en el cual las lactantes y no reproductivas se constituyen en "niñeras" cuando las gestantes salen a alimentarse y viceversa. De manera que, independiente de las cuestiones energéticas y de disminución de competencia que se valoran aquí y por otros autores, en función del tipo de 
refugio que ocupan, el cuidado de las crías durante la actividad nocturna debe resultar de primera importancia para la especie, ya que tanto los que viven en construcciones humanas, como los de cuevas, tienen el mismo comportamiento para garantizar su seguridad. El hecho de que los machos no aparecieran en los grupos de individuos colectados en el sitio de reposo pudiera significar que no participan en el cuidado de las crías, quizás porque no se les permite por las hembras.

\section{Referencias}

AGUIRRE, L.F. 2002. Structure of a neotropical Savanna bat community. Journal of Mammalogy, Baltimore, 83 (3): 775-784

DOLSA, G \& M.T. ALBARRÁN. 1998. La problemática de la contaminación lumínica en la conservación de la diversidad. I sesión de trabajo sobre contaminación lumínica. Departamento de Medio Ambiente, Universidad de Cataluña, Barcelona.

GUNDLACH, J. 1877. Contribución a la mamología cubana. Imprenta G: Montiel, La Habana.

HILL, J. \& J. SMITH. 1984. Bats: A Natural History. Austin: University of Texas Press

MUÑOZ, J. 2001. Los murciélagos de Colombia. Sistemática, distribución, descripción, historia natural y ecología. Editorial Universidad de Antioquia, Medellín.

NOWAK, R. 1997. Velvety free-tailed bat Molosssu molossus. Walker's Mammals of the World, The Johns Hopkins University Press.

SAMPEDRO A, C. M. MARTÍNEZ, K. DE LA OSSA, Y. L. OTERO, L. M. SANTOS. S. OSORIO, \& A. M. MERCADO. 2007. Nuevos registros de especies de murciélagos para el departamento de Sucre y algunos datos sobre su ecología en esta región colombiana. Caldasia, 29 (2):355-362

SAMPEDRO, A., C. MARTíNEZ, A. MERCADO, S. OSORIO, Y. OTERO, L. SANTOS \& R. DÍAZ. 2008a. Refugios, período reproductivo y composición social de las poblaciones de Desmodus rotundus (geoffroy, 1810) (chiroptera: phyllostomidae), en zonas rurales del departamento de Sucre, Colombia. Caldasia, 30(1): 127-134 
SAMPEDRO, A., C. M. MARTÍNEZ, Y. L. OTERO, L. M. SANTOS, S. OSORIO \& A. M. MERCADO. 2008b. Presencia del murciélago casero (Molossus molossus Pallas, 1776) en la ciudad de Sincelejo, departamento de Sucre, Colombia. Caldasia, 30 (2): 495-503

SILVA, G. 1979. Los murciélagos de Cuba. Editorial Academia, La Habana. 423 págs.

ZAR, J. H. (1996): Bioestatistical Analysis. Tercera Edición, Prentice-Hall, Inc., Englewood Cliffs, New Jersey, 718 pp. 